\title{
Clinical Probe of Cyp2C8*2 Mutants in a Malaria Hyperendemic Zone: Evidence from North-Central, Nigeria
}

Olalere Shittu' ${ }^{1, *}$, Olufunke Adenike Opeyemi ${ }^{1}$, Olumuyiwa Babagbemi Omotesho², Oluwatosin Fakayode ${ }^{3}$, Nnaemeka Asogwa4 ${ }^{4}$, Opeyemi Margaret Adeniyi ${ }^{1}$, Ifeoluwa Margaret Fatoba ${ }^{1}$, Kayode Muritala Salawu ${ }^{5}$, Olusola Ajibaye ${ }^{6}$, Olarewaju Abdulkareem Babamale ${ }^{1}$, Oluyinka Ajibola lyiola ${ }^{8}$, Olusola Isaac Aremu ${ }^{7}$

\section{ABSTRACT}

Background: A tremendous level of success has been achieved since the introduction of chloroquine and the combination of amodiaquine and artemisinin for the treatment of both complicated and uncomplicated malaria infections in sub-Saharan Africa. However, the recent discovery of drug resistant strains of Plasmodium falciparum (P. f.) and the ability of the parasite to ingest CYP2C8 into its digestive vacuole is of great public health concern. This study probes the occurrence of CYP2C $8 * 2$ allelic mutant amongst malaria patients in North-Central Nigeria.

Methods: Three hundred and eighty five (385) unrelated study participants were screened for current malaria episodes using routine microscopy and/or rapid diagnostic test strips (RDTs). Chelex extraction method was used for single nucleotide polymorphisms (SNPs) and identification of CYP2C $8 * 2(805 \mathrm{~A}>\mathrm{T})$ variant respectively. Wild-type $(\mathrm{A})$ and the defective allele $(\mathrm{T})$ were differentiated with the use of Polymerase Chain Reaction-Restriction Fragment Length Polymorphism (PCR-RFLP). The results obtained were further validated with Sanger sequencing of a few samples and thereafter, the genotype data were statistically processed. All alleles obtained were in Hardy Weinberg equilibrium.

Results: Out of the 385 participants (45.5\% Male and 54.5\% Female) genotyped for SNPs, 75 (19.5\%) had the autosomal recessive mutant trait. Occurrence of mutant traits was gender and ethnic independent $(p>0.05)$. Yoruba ethnic group recorded a reduction in proportion of genotypic defective CYP2C $8 * 2$ allele ( $T$ ) ( 1 in every 8 persons) with a carrier percentage of $13.3 \%$ compared with Hausa ( $26.62 \%$ ); Igbo (25.37\%) and other minority ethnic groups (17.6\%).

Conclusions: A remarkable inter-ethnic differences in autosomal recessive CYP2C $8 * 2$ allele was observed. By implication, there is a gradual incursion of genetic drift for poor CQ and AQ-Artemisinin metabolizers among the inhabitants.

\section{KEYWORDS}

Plasmodium falciparum; Chloroquine; Amodiaquine-Artemisinin combination therapy; CYP2C8*2; Hausa, Igbo, Yoruba, Nigeria

\section{AUTHOR AFFILIATIONS}

${ }^{1}$ Parasitology Unit, Department of Zoology, University of Ilorin, Ilorin, Nigeria

2 Unilorin Clinic, University of Ilorin, Ilorin, Nigeria

${ }^{3}$ Children Specialist Hospital, Centre Igboro, Ilorin, Nigeria

${ }^{4}$ Department of Biochemistry, University of Ilorin, Ilorin, Nigeria

${ }^{5}$ Department of Pharmacognosy and Drug Development, University of Ilorin, Ilorin, Nigeria

${ }^{6}$ Biochemistry Division, Nigerian Institute of Medical Research, Lagos, Nigeria

7 Department of Pharmaceutics and Industrial Pharmacy, Faculty of Pharmaceutical Sciences, University of Ilorin, Ilorin, Nigeria

${ }^{8}$ Cell Biology and Genetics Unit, Department of Zoology, University of Ilorin, Ilorin, Nigeria

* Corresponding author: Parasitology Unit, Department of Zoology, University of Ilorin, Ilorin, Nigeria; e-mail: eternity403@yahoo.com

Received: 24 September 2019

Accepted: 22 June 2020

Published online: 1 October 2020

Acta Medica (Hradec Králové) 2020; 63(3): 119-123

https://doi.org/10.14712/18059694.2020.29

(c) 2020 The Authors. This is an open-access article distributed under the terms of the Creative Commons Attribution License (http://creativecommons.org/licenses/by/4.0), which permits unrestricted use, distribution, and reproduction in any medium, provided the original author and source are credited. 


\section{INTRODUCTION}

Infections arising as a result of Plasmodium falciparum is the major cause of malaria-related deaths and it has been reported to be the most common of the four human malaria parasites across sub-Saharan Africa (1). Despite wide documented chloroquine (CQ) and Amodiaquine (AQ) resistance; majority of the populace still rely on therapeutic $\mathrm{CQ}$ and $\mathrm{AQ}$ medications. The increasing failure of these drugs against falciparum malaria constitutes a notable setback in the eradication efforts of malaria in many African countries (2-4). Previous studies have established that host genetic variations with respect to cytochrome P450 (CYP) 2C8 (CYP2C8) as drug metabolizers is responsible for the metabolism of about $20-50 \%$ clinical drugs and endogenous substances. The emerging mutations with respect to these metabolites is one of the main risk factors for the drug resistant strains of P. falciparum in Africa (5). Genotype-inferred low metabolizers were reported in $1-4 \%$ of African populations corresponding to millions of expected exposures to AQ (6). Recent studies further revealed that resistant strain are often characterized with the genetic defective variant (CYP2C $\left.{ }^{*} 2\right)$ identified as being responsible for the hepatic metabolism of $C Q$ and $A Q$, consequently altering chloroquine flux or reduced drug binding to hematin inside the parasite digestive vacuole. This mechanism culminates into Plasmodium falciparum chloroquine resistance transporter (PfCRT) point mutations $(5,7)$. Similarly, quinoline ring in $4 \mathrm{Q}$ s is resistant to degradation by cytochrome P450 enzymes (CYP) CYP2C8 and CYP3A4, with potentials to mediate $80 \%$ of the total metabolism of $4 \mathrm{AQs}$ (8). However, the common occurrence of genetic variant CYP2C $8 * 2$ in malaria infected host has been linked to the presence of drug-resistant parasites in the infected host (pfcrt-76Y and pfmdr1-86Y P. falciparum alleles). This anomaly is documented as a strong factor that is chronically hindering the efficacy of CQ and AQ in Africa. A recent longitudinal study in Africa reported that the prevalence of the defective allele "CYP2C 8 * 2 " is statistically insignificant among ethnic groups in Nigeria, although comparable with what was obtained in Senegal and Madagascar (9). However, it is pertinent to further explicate the presence of this allele in other African descents, because of the crucial role it plays in the epidemiology of falciparum infections. This study will investigate the occurrence and determine the allele frequencies of CYP2C $8 * 2$ amongst residents in a malaria high transmission zone of North-Central Nigeria. This is to guide intervention for better understanding of the metabolic mechanism of $\mathrm{CQ}$ and $\mathrm{AQ}$ artemisinin-based combination therapy (ACT) in the study area.

\section{MATERIALS AND METHODS}

\section{STUDY AREA, DESIGN AND PROTOCOLS}

The study was conducted within Ilorin metropolis, an urban area, in the North-Central zone and the capital of Kwara State, Nigeria. It is located on longitude $4^{\circ} 35^{\prime} \mathrm{E}$ and latitude $8^{\circ} 35^{\prime} \mathrm{N}$. It covers an area of about 38 square miles, with an estimated population of 1.4 million people. The area is associated with intense rainfalls from April to October and daily temperature of between $23^{\circ} \mathrm{C}$ and $37^{\circ} \mathrm{C}$. Inhabitants are mostly farmers, civil servants, traders and students. Out-patients from four randomly selected hospitals (Civil Service Hospital, Temitope Hospital, Children Specialist Hospital and University of Ilorin Health Centre) in Ilorin were used for the study. A simple structured questionnaire was administered to volunteers after written informed consent was sought and approved to obtain some basic information on ethnicity and Knowledge about usage of antimalarials like CQ, AQ and ACTs (viz; Artemether-lumefantrine, Artesunate-mefloquine and Dihydroartemisinin-piperaquine). Only volunteers with a record of past $C Q$ and $A Q$ medications were considered for the present study. Intravenous blood samples of subjects were spotted on Whatman number 3 filter papers, air dried and separately stored in sealed plastic containers. Routine malaria diagnosis was initially performed by microscopic examination of Giemsa-stained thick blood smears and/ or a rapid diagnostic test (Malaria Antigen P. f., Standard diagnostics, INC. Ingbert, Germany). Single nucleotide polymorphisms in CYP2C $8 * 2$ was screened for according to Marwa et al. (10). DNA extraction and subsequent identification of CYP2C $8 * 2(805 \mathrm{~A}>\mathrm{T})$ variant was carried out using Chelex extraction method and Polymerase Chain Reaction-Restriction Fragment Length Polymorphism (PCR-RFLP) respectively as described by Paganotti et al. (11). Briefly, $2 \mu$ of DNA template was amplified by PCR, at $107 \mathrm{bp}$ fragment for the CYP2C8 gene forward primer (i.e. at 5'-GAACACCAAGCATCACTGGA-3') and reverse primer (i.e. at $5^{\prime}$-GAAATCAAAATACTGSTCTGTTGC-3'). The products from PCR analysis was incubated with $\mathrm{Bcl} 1$ enzyme that cuts the wild type allele only (A); undigested products then represent the variant allele $(\mathrm{T})$. In order to detect the size polymorphisms, both types were allowed to run on a metaphor 3\% gel. Controls for human genotyping were then utilized after sequencing of the PCR product obtained from each different genotype. Genotyping errors were avoided by double checking for the heterozygous samples.

\section{ETHICAL APPROVAL}

This study was performed according to the Declaration of Helsinki and the procedure followed was part of a study approved by the University of Ilorin Ethical Consideration with protocol approval number: UERC/ASN/2012/221. Consent form was administered and collected from the volunteers before the commencement for the study.

\section{STATISTICAL ANALYSIS}

Data obtained were analyzed with SigmaPlot for Windows version 12.0 (Systat. Software, Inc.). The prevalence of recorded alleles (i.e. wild and mutant alleles) were subjected to Chi-square $(\chi 2)$ analysis and statistical significance was set at $p<0.05$. The results obtained were further validated with Sanger sequencing of a few samples and thereafter, the genotype data were statistically processed. Hardy-Weinberg equation $\left(\mathrm{p}^{2}+2 \mathrm{pq}+\mathrm{q}^{2}=1\right)$ was used to estimate the frequency of the carrier state $(2 \mathrm{pq})$ for autosomal recessive trait among the study population (12). 
Tab. 1 Characteristics of samples taken for CYP2C $8 * 2$ analysis.

\begin{tabular}{|c|c|c|c|c|}
\hline \multirow[t]{2}{*}{ Factor } & \multirow[t]{2}{*}{ No examined (\%) } & \multicolumn{3}{|c|}{ Allele frequency (\%) } \\
\hline & & Mutants & Wild Type & p-value \\
\hline Total no examined & 385 & 75 (19.5) & 310 (80.5) & \\
\hline \multicolumn{4}{|l|}{ Gender } & 0.814 \\
\hline Male & $175(45.5)$ & $35(46.7)$ & $140(45.2)$ & \\
\hline Female & $210(54.5)$ & $40(53.3)$ & $170(54.8)$ & \\
\hline \multicolumn{4}{|l|}{ Age group } & 0.003 \\
\hline $0-5$ & $100(26.0)$ & $20(26.7)$ & $80(25.8)$ & \\
\hline $6-15$ & $90(23.4)$ & $10(13.3)$ & $80(25.8)$ & \\
\hline $16-25$ & $50(13.0)$ & $20(26.7)$ & $30(9.7)$ & \\
\hline $26-35$ & 35 (9.1) & $5(6.7)$ & $30(9.7)$ & \\
\hline $36-45$ & $60(15.6)$ & $10(13.3)$ & $50(16.1)$ & \\
\hline$>45$ & $50(13.0)$ & $10(13.3)$ & $40(12.9)$ & \\
\hline \multicolumn{4}{|l|}{ Ethnic group } & 0.102 \\
\hline Yoruba & $195(50.6)$ & $45(60.0)$ & $150(48.4)$ & \\
\hline Hausa & $40(10.4)$ & $10(13.3)$ & $30(9.7 \%)$ & \\
\hline Igbo & 45 (11.7) & $5(6.7)$ & 40 (12.9) & \\
\hline Others* & $105(29.9)$ & $15(20.0)$ & $90(29.0)$ & \\
\hline
\end{tabular}

* Idoma (30, 7.8\%), Fulani (10, 2.6\%), Nupe (40, 10.4\%), Igala (10, 2.6\%) and Benue/Igede (15, 3.9\%).

\section{RESULTS}

Three hundred and eighty five (385) individuals consisting of 175 (45.5\%) male and 210 (54.5\%) female with a past record of CQ and AQ use voluntarily participated in this study. Seventy five (75 (19.5\%)) was analysed to have recessive mutant traits of CYP2C8*2 allele. Mutant population with respect to gender and ethnic group were not statistically significant $(\mathrm{p}>0.05)$. However, the defective CYP2C ${ }^{*} 2$ allele in comparison with the wild dominant allele was significant with respect to distribution among the respective age groups sampled $(\mathrm{p}=0.003)$ (Table 1$)$.

The genotype and allele frequencies in the Nigerian major and minor ethnic groups domicilied in the study area was assessed with Hardy-Weinberg equilibrum calculator (12). The genotype frequencies obtained obeyed the assumptions layed down for the principle. For instance, the allele frequency for this generation was done by pooling together the alleles from each genotype of the same generation according to the expected contribution from the homozygote and heterozygote genotypes. Yoruba ethnic group recorded a reduction in proportion of CYP2C $8 * 2$ allele $(\mathrm{T})$ frequency ( 1 in every 8 persons) with a carrier percentage of $13.3 \%$ despite the large sample size screened ( $\mathrm{N}=195)$ compared with others (viz; Hausa: 26.62\%; Igbo: 25.37\%; Others: $17.6 \%$ ) (Table 2).

\section{DISCUSSION}

The recent discovery of molecular markers for drug resistance in genomic studies is gradually eliciting various dimension (13). Investigation on defective CYP2C $8 * 2$ is essential to evaluate emergence of antimalarial drug resistance markers ( $P$. falciparum) population among the three major Nigerian ethnic groups. In this study, a non-negligible frequency (19.5\%) of autosomal recessive CYP2C8*2 mutants was obtained among malaria patients. This outcome is similar to the report of Adehin et al. (14) in South-west Nigeria. However, this study reported a lower

Tab. 2 Genotypes for CYP2C8*2 and T allele frequency among the studied Ethnic groups.

\begin{tabular}{|l|l|l|l|l|l|l|}
\hline \multirow{2}{*}{ Ethnic groups } & \multicolumn{2}{l}{ CYP2C8*2 (rs11572103, A > T) } & \multicolumn{3}{l|}{ Allele frequency } \\
\cline { 2 - 7 } & Genotype frequencies & AA & AT & TT & T & Carrier (\%) \\
\cline { 2 - 7 } & N & 0.862 & 0.133 & 0.005 & 1 in 8 & $26(13.3)$ \\
\hline Yoruba & 195 & 0.709 & 0.266 & 0.025 & 1 in 4 & $11(26.62)$ \\
\hline Hausa & 40 & 0.724 & 0.254 & 0.022 & 1 in 4 & $11(25.37)$ \\
\hline Igbo & 45 & 0.814 & 0.176 & 0.010 & 1 in 6 & $18(17.61)$ \\
\hline Others* & 105 & & &
\end{tabular}

* Idoma (30, 7.8\%), Fulani (10, 2.6\%), Nupe (40, 10.4\%), Igala (10, 2.6\%) and Benue/lgede (15, 3.9\%). AA-homozygous wild-type; AT-heterozygous carrier; TT-homozygous mutant; T-Phenotype. 
prevalence of $\mathrm{CYP} 2 \mathrm{C} 8 * 2$ status when compared with several early studies reports in African populations $(10,11,15$, 16). In a similar vein, CYP $2 \mathrm{C} 8^{*} 2$ allele was successfully genotyped in $75 \%$ (213/285) of children in Congo Brazzaville. The CYP2C $8 * 2 \mathrm{~A}$ allele had a frequency of $63 \%$, whereas the CYP2C ${ }^{*} 2 \mathrm{~T}$ allele had a frequency of $37 \%$. Genotypes CYP2C $8 * 2 \mathrm{AA}$ (rapid metabolizer), CYP2C $8 * 2 \mathrm{AT}$ (intermediate metabolizer), and CYP2C $8 * 2 \mathrm{TT}$ (poor metabolizer) were reported in $44 \%, 38 \%$, and $18 \%$ of the investigated participants, respectively (17). This suggests that mutations in specific $P$. f. genes may confer resistance to antimalarial drugs, climaxing into sustained drug pressure (18). Also, this finding may serve as an important tool at predicting the level of resistance to $C Q$ and $A Q+$ Artemisinin combinations drugs. It is suffice to mention that in sub-Saharan Africa, people carrying CYP2C ${ }^{*} 2$ C.805A $>\mathrm{T}$ (CYP2C8*2; rs11572103) allele suffer impaired amodiaquine metabolism, increased risk of amodiaquine-related adverse events, and may promote the selection of drug-resistant parasite strains (17). CYP2C8 accounts for the metabolism of $>20 \%$ of drugs used in the treatment of varying ailments with over 60 clinically important therapeutic agents of which malaria is one (19). However, CYP2C $8^{*} 2 \mathrm{~T}$ allele occurs mostly in people with a sub-Saharan Africa ancestry $(19 \%)$ and it is less frequent $(\leq 1 \%)$ in individuals of European, Asian, or American origin (20). The 4-Qs become resident in the acidic digestive vacuole, where they are believed to bind b-hematin and interfere with heme detoxification (21). In human, AQ is mainly metabolized in the liver, and CYP2C8 is the main hepatic isoform that catalyzes the formation of $\mathrm{N}$-desethylaminodiaquine (DEAQ) (22). From the aforementioned, it is obvious that the defective allele give rise to a number of different point mutations affecting the heme and or substrate binding ability of CYP2C8 as it is the most abundant form expressed in the liver and other extrahepatic tissues (23). In our study, genotypic data obtained showed that the Yoruba ethnic group' chances of outcome with the defective allele was one in eight which appears to be the least because the carrier frequency was $13.3 \%$, amongst the studied population. The observed variance may be due to the differences in population sampled or it may be ascribed to activity impacting nature of the SNPs playing less relevance in some descents $(10,14)$. CYP2C8 also metabolizes arachidonic acid and the anticancer drug paclitaxel, and CYP2C8 variants have been shown to be defective in the metabolism of both substrates (22). The emergence and spread of drug resistance depends, in part, on the number of mutations required to encode resistance and their effects on parasite fitness (24). Specific multiple point mutations is however very important in a gene make up of a resistant marker for an antimalarial drug $(20,25)$. Many adverse reactions are attributable to reduced CYP2C8 expression, but yet unreported during clinical trials. The expression often leads to any one or all of the following, viz; poor metabolizer phenotypes, hepatoxicity and a severe reduction in white blood cell count (22). The aforementioned may result in the risk of both mild and severe adverse clinical outcomes associated with AQ treatment (16). Furthermore, the presence of this defective allele in our population is suggestive of possible inter-population differences in clinical outcomes associated with ACT drugs.

\section{CONCLUSION}

Currently, the increasing knowledge in genomic revolution is significantly improving our understanding of reasons why individuals and populations differ in their susceptibility to multiple diseases. The occurrence of inter-ethnic differences in the frequencies of clinically relevant CYP450 variants is the real reason populations don't maintain the stable allele frequencies predicted by the Hardy-Weinberg equilibrium. A remarkable inter-ethnic differences in autosomal recessive CYP2C $8 * 2$ allele was observed. By implication, there appears to be a gradual incursion of genetic drift for poor CQ and AQ-Artemisinin metabolizers among the inhabitants. Further studies are required to evaluate the toxicological significance of this poor metabolizer in our settings and give insights to the adverse effect on the drug pharmacokinetics of $C Q$ and AQ-ACT drugs.

\section{AUTHORS CONTRIBUTION}

OSH, OAB, OIA and MKS designed, did statistical analysis and contributed to the manuscript write-up, OAO supervised the collection of the samples, $\mathrm{OBO}$ and $\mathrm{OF}$ provided patients in their respective hospitals, OMA and MIF collected dried blood spot samples, AE, OAI and AO carried out the PCR and RFLP analysis. OIA edited the final manuscript.

\section{COMPETING INTEREST}

The authors of this study declare no competing interest. All authors partook in the design, implementation and the write-up.

\section{REFERENCES}

1. Jamison D, Feachem R, Makgoba M, et al.: The International Bank for Reconstruction and Development. Washington (DC): The World Bank 2006.

2. Petersen I, Eastman R, Lanzer M. Drug-resistant malaria: Molecular mechanisms and implications for public health. FEBS Letters 2011; 585(11): 1551-62.

3. Kumar SC. Drug Resistance in Malaria. In: Drug Resistance in Bacteria, Fungi, Malaria, and Cancer. Springer 2017: 429-47.

4. Antony HA, Parija SC. Antimalarial drug resistance: An overview. Tropical Parasitology 2016; 6(1): 30 .

5. Paganotti GM, Gallo BC, Verra F, et al. Human genetic variation is associated with Plasmodium falciparum drug-resistance. J Infect Dis 2011; 204: 1772-8.

6. Gil JP, Gil BE. CYP2C8 and antimalaria drug efficacy. Pharmacogenomics 2007; 8(2): 187-98.

7. Parkinson A, Kazmi F, Buckley DB, Yerino P, Ogilvie BW, Paris BL. System-dependent outcomes during the evaluation of drug candidates as inhibitors of cytochrome P450 (CYP) and uridine diphosphate glucuronosyltransferase (UGT) enzymes: human hepatocytes versus liver microsomes versus recombinant enzymes. Drug Metabolism and Pharmacokinetics 2010; 25(1): 16-27.

8. Kalia S, Dutz JP. New concepts in antimalarial use and mode of action in dermatology. Dermatologic therapy 2007, 20(4):160-174.

9. Adehin A, Bolaji OO, Kennedy MA. Polymorphisms in CYP2C8 and CYP3A5 genes in the Nigerian population. Drug Metabolism and Pharmacokinetics 2017; 32(3): 189-91. 
10. Marwa KJ, Schmidt T, Sjögren M, Minzi OMS, Kamugisha E, Swedberg G. Cytochrome P450 single nucleotide polymorphisms in an indigenous Tanzanian population: a concern about the metabolism of artemisinin-based combinations. Malaria Journal 2014; 13: 420.

11. Paganotti GM, Gramolelli S, Tabacchi F. Distribution of human CY P2C8*2 allele in three different African populations. Malaria Journal 2012; 11: 125

12. Carrier Frequency Calculator (http://www.perinatology.com/calcu lators/Hardy-Weinberg.htm).

13. Lin JT, Juliano JJ, Wongsrichanalai C. Drug-Resistant Malaria: The Era of ACT. Current Infectious Disease Report 2010; 12(3): 165-73.

14. Adehin A, Bolaji OO, Kennedy MA. Polymorphisms in CYP2C8 and CYP3A5 genes in the Nigerian population. Drug Metabolism and Pharmacokinetics 2016; 30: 1-3.

15. Fernandez P, Zeigler-Johnson CM, Spangler E, et al.: Androgen metabolism gene polymorphisms, associations with prostate cancer risk and pathological characteristics: a comparative analysis between south african and senegalese men. Prostate Cancer 2012: 798634.

16. Bains RK. African variation at Cytochrome P450 genes: Evolutionary aspects and the implications for the treatment of infectious diseases. Evolution, Medicine, and Public Health 2013; 2013(1): 118-34.

17. Peko SM, Ntoumi F, Vouvoungui C. Distribution of the cytochrome P450 CYP2C8*2 allele in Brazzaville, Republic of Congo. International Journal of Infectious Diseases 2019, 85: 49-53.
18. White NJ. Malaria. Edinburgh, United Kingdom: Elsevier Science Limited 2003.

19. VandenBrink BM, Foti RS, Rock DA, Wienkers LC, Wahlstrom JL. Evaluation of CYP2C8 inhibition in vitro: utility of montelukast as a selective CYP2C8 probe substrate. Drug Metabolism and Disposition 2011; 39: 1546-54.

20. Backman JT, Filppula AM, Niemi M, Neuvonen PJ. Role of Cytochrome P450 2C8 in Drug Metabolism and Interactions. Pharmacological Reviews 2016; 68(1): 168-241.

21. Greenwood BM, Fidock DA, Kyle DE, et al. Malaria: progress, perils, and prospects for eradication. Journal of Clinical Investigation 2008; 118(4): 1266-76.

22. Dai D, Zeldin DC, Blaisdell JA, et al. Polymorphisms in human CYP2C8 decrease metabolism of the anticancer drug paclitaxel and arachidonic acid. Pharmacogenetics 2001; 11: 597-607.

23. Gil JP, Gil Berglund E. CYP2C8 and antimalaria drug efficacy. Pharmacogenomics 2007; 8(2): 187-98.

24. White N. Antimalarial drug resistance and combination chemotherapy. Philosophical Transactions of the Royal Society of London Series B, Biological Sciences 1999: 354: 739-49.

25. Agomo CO, Oyibo WA, Sutherland C, Hallet R, Oguike M. Assessment of Markers of Antimalarial Drug Resistance in Plasmodium falciparum Isolates from Pregnant Women in Lagos, Nigeria. PloS One 2016; 11(1): e0146908-e0146908. 